\title{
IMPLANTAÇÃO DE UM SISTEMA PRODUTO-SERVIÇO EM UMA EMPRESA DE MANUFATURA: DIFICULDADES OBSERVADAS
}

Antonio Erlindo Braga Jr. (erlindo@uepa.br) - Departamento de Desenho Industrial, Universidade do Estado do Pará.

José Carlos de Toledo (toledo@dep.ufscar.br) - Departamento de Engenharia de Produção, Universidade Federal de São Carlos.

\section{RESUMO}

A implantação de um Sistema Produto-Serviço (PSS) em empresas de manufatura visa o alcance dos benefícios esperados e amplamente divulgados na literatura científica. Entretanto, verifica-se nas publicações casos em que, apesar dos esforços para incorporar serviços nos negócios de uma empresa de manufatura, não se alcança o retorno financeiro que justifique estes investimentos. Assim, o processo de implantação de um PSS não está livre de ser simples e óbvio. Para uma melhor taxa de sucesso nesta atividade é necessário obter maior conhecimento sobre os obstáculos existentes no processo de implantação de um PSS e, para tanto, este artigo identifica as dificuldades observadas durante este processo em uma empresa de manufatura no Brasil. Os resultados do trabalho permitiram confirmar o já observado anteriormente na literatura, mas também gerou novas contribuições. Verificou-se a existência de dificuldades não relatadas na bibliografia e referentes à preparação da empresa, mais especificamente sobre o treinamento dos funcionários e a obtenção de maior conhecimento sobre as particularidades do PSS. Observou-se que as dificuldades no processo de implantação são também causadas pelo método escolhido para o desenvolvimento do PSS. Os resultados da pesquisa contribuem para que futuros desenvolvedores de PSS previnam-se mais adequadamente aos óbices deste processo.

Palavras-chave: Sistema Produto-Serviço, PSS, implantação de PSS, dificuldades.

Área: Desenvolvimento de Sistemas Produto-Serviço (PSS).

\section{INTRODUÇÃO}

O Sistema Produto-Serviço, ou Product-Service Systems (PSS) traz vantagens para as empresas que o adotam como a maior competitividade, a redução dos custos com a produção de bens de consumo, melhor relação com o usuário e maior satisfação das suas necessidades, aumento na produção de empregos e redução dos impactos ambientais, são alguns dos exemplos observados (CAVALIERI; PEZZOTTA, 2012; DIMACHE; ROCHE, 2013; GOEDKOOP et al., 1999; KOHTAMÄKI et al., 2015; MATHIEU, 2001). Contudo, alguns fatores impedem a ampla disseminação deste sistema na indústria de manufatura.

Alguns gestores de empresas são relutantes na transição para o PSS por entenderem que há a necessidade de mudanças fundamentais na cultura organizacional, no marketing, na produção e um alto investimento em infraestrutura (DIMACHE; ROCHE, 2013). Outros enfrentam dificuldades na concepção, desenvolvimento e entrega do PSS, na implantação das mudanças necessárias na cultura empresarial e nos setores da empresa (VEZZOLI; KOHTALA; SRINIVASAN, 2014). 
Apesar das premissas de geração de valor e vantagem competitiva, o impacto dos resultados da estratégia nos serviços é incerto. Enquanto algumas empresas possuem sucesso com a servitização outras lutam para obter um desempenho financeiro satisfatório (BAINES et al., 2007). Gebauer, Fleisch e Friedli (2005) verificaram que apesar de algumas empresas de manufatura investirem fortemente para incorporar serviços em seus negócios, e incorrer em custos de operação mais elevados, isso não tem se revertido em retorno financeiro que justifique estes investimentos. Estes autores denominaram este fato de "paradoxo de serviços em empresas de manufatura". Na figura 1, situação 1, a empresa de manufatura explora o potencial financeiro da expansão para a oferta de serviços, recebendo a resposta esperada aos investimentos realizados, ou seja o retorno satisfatório por unidade monetária investida. $\mathrm{Na}$ situação 2, a empresa de manufatura realiza os investimentos para a ampliação da oferta de serviços, mas não gera o retorno esperado, gerando baixa rentabilidade em relação ao capital investido.

Figura 1. Paradoxo de serviços. Fonte: Gebauer, Fleisch e Friedli (2005)

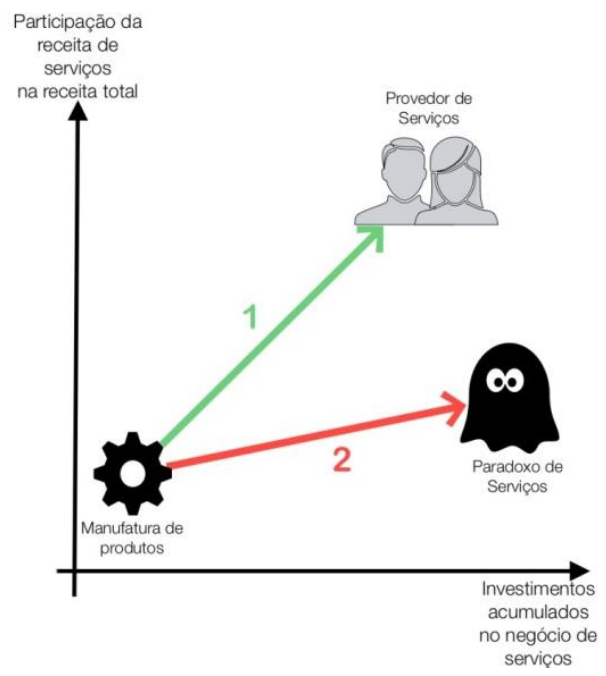

De acordo com estes autores há diversas barreiras que impedem as empresas de manufatura de acessarem os benefícios de um PSS e são oriundas de duas fontes principais: a primeira é de ordem cognitiva que dificulta que gestores mudem sua forma de ver o negócio e a segunda está ligada às diversas mudanças necessárias na estrutura organizacional das empresas de manufatura.

\section{DIFICULDADES NA IMPLANTAÇÃO DE SISTEMAS PRODUTO-SERVIÇO OBSERVADAS NA BIBLIOGRAFIA}

Braga Jr. e Toledo (2016) realizaram um levantamento bibliográfico em que identificaram sete classes de dificuldades para o desenvolvimento e implantação de Sistemas ProdutoServiço em empresas de manufatura:

- Dificuldades relativas ao Marketing - referentes às práticas de marketing da empresa ou ligadas ao antigo modelo de oferta unicamente de produtos;

- Dificuldades organizacionais - relacionadas às mudanças na cultura empresarial voltada a negócios orientados para serviços;

- Dificuldades relativas ao projeto do produto e do serviço - há o aumento da complexidade desta tarefa pela necessidade de reunir e organizar as várias partes interessadas na concepção tanto do produto quanto do serviço. Há o risco de aumento 
do tempo de lançamento do produto no mercado devido ao aumento e diversidade dos requisitos de informação para a concepção de um PSS;

- Dificuldades relativas ao relacionamento com clientes e fornecedores - a empresa fornecedora do PSS precisa desenvolver sistemas de suporte de serviços e sistemas para monitorar a condição dos produtos nas instalações dos usuários sem que sejam considerados invasivos às atividades do cliente;

- Dificuldades relativas à cultura organizacional - alterar a orientação da empresa de produto para serviço implica na mudança na cultura corporativa e o engajamento no mercado de atuação, o que, por sua vez, demanda tempo e recursos para a sua viabilização;

- Dificuldades relativas ao alinhamento estratégico - são referentes aos desafios na nova definição da estratégia da organização e na sua transformação;

- Outros tipos de dificuldades - relacionadas à quantificação dos benefícios oriundos do PSS em termos econômicos e ambientais, falta de conhecimento sobre o projeto eficiente do PSS e a necessidade de formação de uma nova geração de projetistas e de pessoal para formar estes profissionais.

\section{MÉTODO DE PESQUISA}

A pesquisa se deu seguindo a estrutura de uma Pesquisa-Ação cujo característico é o desenvolvimento simultâneo de atividades (ação) e de coleta de dados (pesquisa) para a sua posterior análise. O processo se deu com o desenvolvimento do processo de implantação do Sistema Produto-Serviço e da observação e registro das dificuldades para o desenvolvimento deste processo. A Pesquisa-Ação é composta pela etapa preparatória, etapas principais e etapa de monitoramento (COUGHLAN; COGHLAN, 2002).

A etapa preparatória teve como objetivo o melhor entendimento do negócio da empresa, de sua cultura de trabalho assim como sensibilizar os participantes no processo de implantação do PSS para a importância da sua atuação nas discussões e na execução das tarefas.

As etapas principais são compostas pelas sub-etapas "Coleta de Dados", "Feedback de Dados", "Análise dos Dados", "Planejamento das Ações", "Implantação" e "Avaliação" e caracterizam um ciclo da Pesquisa-Ação. Cada ciclo foi definido como uma fase do método de desenvolvimento de Sistemas Produto-Serviço proposto por Vezzoli Kohtala e Srinivasan (2014). Este método é caracterizado por uma estrutura modular e flexível, de modo que pode ser adaptado às necessidades específicas das empresas, para diversos contextos e condições de projeto. A estrutura básica do método de desenvolvimento do PSS consiste em quatro fases principais: Análise Estratégica, Levantamento de Oportunidades, Projeto de Conceitos do Sistema e Detalhamento do Sistema.

Para a adequada execução das etapas principais da Pesquisa-Ação com as fases do método de desenvolvimento do PSS estabeleceu-se como objetivo de cada ciclo o objetivo das fases. Portanto, para cada fase do processo de desenvolvimento do PSS foi cumprido um ciclo da Pesquisa-Ação como é apresentado na figura 2.

A etapa de monitoramento é uma "meta-etapa", pois ocorre durante todos os ciclos da pesquisa-ação. Cada ciclo deverá levar a um novo ciclo que irá repetir todas as etapas descritas anteriormente, como mostrado na figura 3. Foi realizada de forma simultânea com as demais etapas e realizou o acompanhamento das atividades realizadas assim como verificou o cumprimento das tarefas e responsabilidades de cada fase do ciclo da Pesquisa-Ação. Nesta 
etapa a ação é observada para a coleta de evidências que fornece a base para a reflexão e onde foram realizadas as observações das dificuldades de implantação do PSS. Como consequência, esta etapa gerou dados para a análise e discussão das dificuldades para a implantação do PSS na empresa.

Figura 2. Execução dos ciclos da Pesquisa-Ação e das fases do método de desenvolvimento de PSS. Fonte: Os autores.

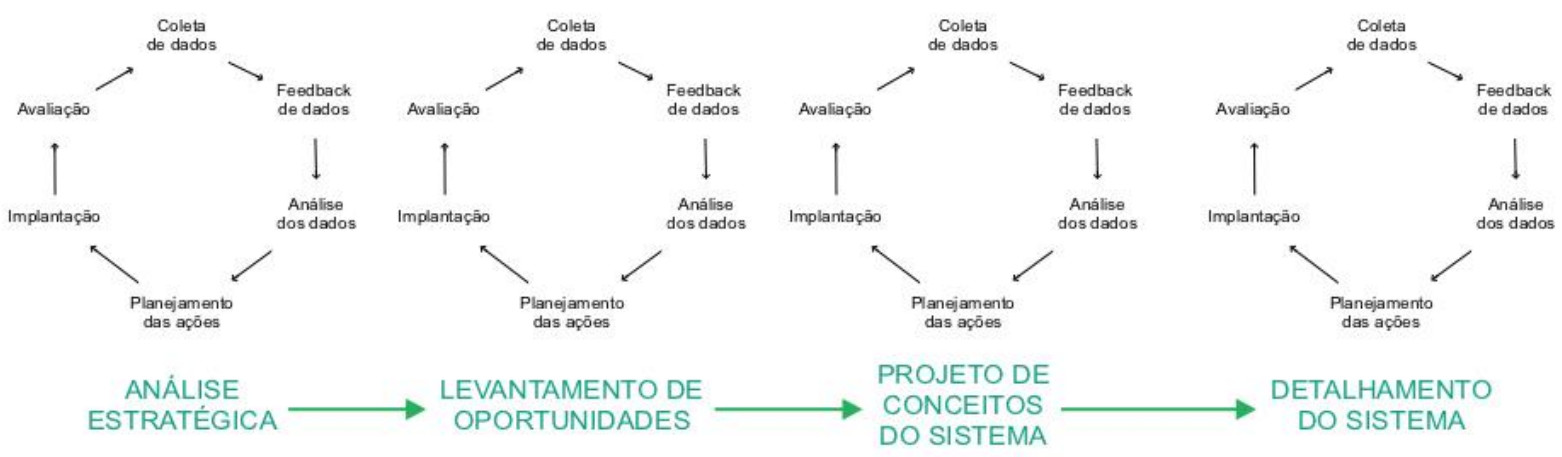

Figura 3. Estruturação da Pesquisa-Ação. Fonte: Coughlan e Coghlan (2002)

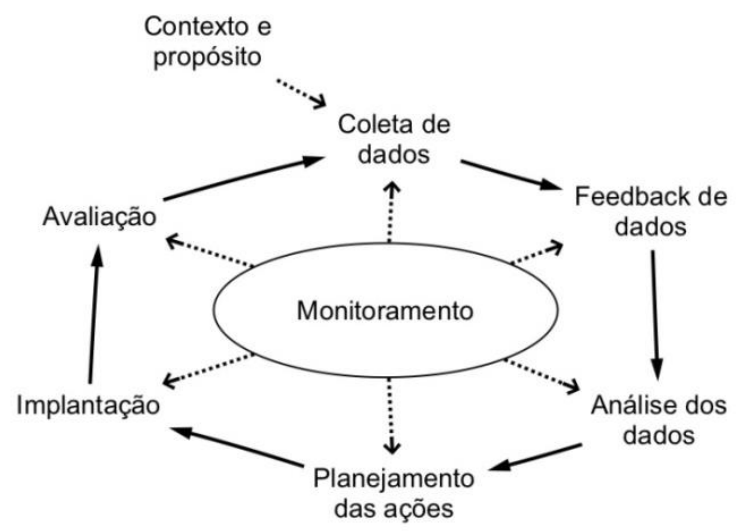

\section{DIFICULDADES OBSERVADAS NO PROCESSO DE IMPLANTAÇÃO DO SISTEMA PRODUTO-SERVIÇO}

O Sistema Produto-Serviço foi implantado em uma empresa do setor da Construção Civil (pré-fabricados em concreto) e foram observados dois tipos de dificuldades: as relacionadas à empresa e as relacionadas ao método escolhido para implantação do PSS, apresentadas nos subitens a seguir.

\section{1 - Dificuldades observadas junto à empresa}

No início das atividades de implantação do PSS foi feita uma entrevista com o proprietário da empresa e perguntou-se quais seriam as dificuldades que o mesmo visualizava na realização do trabalho. A ideia com este questionamento foi identificar alguns anseios do empresário como também eventuais dificuldades não identificadas anteriormente na bibliografia. As potenciais dificuldades e anseios observados nesta entrevista são os seguintes:

- Necessidade de treinamento específico da equipe para o entendimento do novo posicionamento estratégico da empresa;

- Em uma empresa focada em produtos muitos problemas não são percebidos porque o 
cliente vê apenas o resultado final, não toma conhecimento dos ajustes realizados no processo. Em serviços o processo é mais explícito para o cliente;

- Preocupação quanto à existência de uma barreira no mercado para o entendimento e aceitação deste novo modelo de negócios da empresa.

Em relação à equipe delegada para o desenvolvimento do trabalho verificou-se um descrédito inicial dos seus participantes em relação ao sucesso do PSS. Acredita-se que as causas deste comportamento foram por conta da novidade do tema para os participantes, das possíveis mudanças de procedimentos que já se vislumbrava, da repercussão destas mudanças para a estratégia da empresa e dos negócios e da desconfiança gerada em virtude do condutor do trabalho ser um elemento externo à empresa.

Ainda em relação à equipe de desenvolvimento, foi observada dificuldade na compreensão da mudança para o novo posicionamento da empresa. Alguns participantes do grupo entendiam que se deveria buscar maior redução de custos no processo de produção e, por conta disso, deveria haver investimento em equipamentos ao invés de se realizar a implantação do PSS. Em rebate a esta posição, o pesquisador ressaltou que o diagnóstico realizado pelo próprio grupo na etapa preparatória da Pesquisa-Ação concluía que a empresa deveria buscar a diferenciação e que já havia diversas empresas no mercado que procuravam produção em larga escala combinado com baixo preço. Houve ainda uma barreira cultural para o entendimento da oferta combinada entre produtos e serviços, alguns participantes demandaram um tempo maior para entender como se daria a nova proposta de valor da empresa.

Outra barreira observada foi a dificuldade em garantir o cumprimento das atividades planejadas na Pesquisa-Ação. Como este método de pesquisa demanda uma maior participação dos membros da empresa esta barreira tornou-se mais importante que as demais observadas. Para isto foi necessário demonstrar à equipe a importância do trabalho para a empresa assim como conquistar a confiança dos participantes para garantir que as horas dedicadas para as discussões não se tornassem irrelevantes.

O desconhecimento dos conceitos básicos do PSS certamente foi um impeditivo para que o trabalho não se desenvolvesse de maneira mais célere. A mudança do modelo de negócios da empresa, a falta de visão e conhecimento de alternativas de modelos de negócios também contribuíram para o desenvolvimento mais lento das atividades. É importante ressaltar que esta barreira foi sendo contornada à medida que as reuniões ocorriam e se tornou possível explanar e entender os conceitos que motivavam a mudança na empresa assim como os conceitos básicos sobre o PSS.

Houve problemas também em relação ao agendamento de horários de reunião assim como de cumprimento deste agendamento. Em virtude da implantação do PSS não ser o cerne do trabalho da equipe que estava envolvida na Pesquisa-Ação havia uma relativa dificuldade em agendar as reuniões e o seu cancelamento ocorreu em algumas oportunidades sem uma comunicação prévia. Outro fator que influenciou nestes problemas foi a demanda pelo atendimento de pedidos de clientes por orçamentos, projetos ou outra atividade referente ao escopo previsto pela equipe de engenheiros da empresa.

A Pesquisa-Ação prevê a participação das pessoas da empresa de forma ativa. Algumas das atividades repassadas não eram cumpridas, havendo a necessidade de se adiar alguns encontros, ou ainda atrasando o início da reunião, aguardando-se que a tarefa fosse realizada para que o objetivo estabelecido fosse cumprido.

Observou-se um receio de que eventuais modificações poderiam aumentar o custo fixo da empresa, como apresentado na figura 1, gerando o "Paradoxo de Serviços". Este temor foi 
potencializado pela situação financeira que a empresa passava à época do desenvolvimento do trabalho e pela recessão econômica do país. E por fim, em virtude dos problemas financeiros apontados, observou-se que a gestão da empresa não tinha pleno foco no trabalho de implantação do PSS apesar de estar sensibilizada para a mudança necessária no modelo de negócios da empresa. Esta barreira foi reforçada por uma característica comportamental deste empreendedor, o qual possui perfil de atuação predominantemente operacional e pouco estratégico.

\section{2 - Dificuldades observadas junto ao método escolhido}

Com a implantação do PSS na empresa de manufatura observou-se que o método não é completo e precisa ainda de melhorias para detalhar as funções da equipe, especificar procedimentos e interações na operação do PSS. Para a melhor organização das dificuldades observadas, estas serão apresentadas em função das etapas de desenvolvimento determinadas pelo método utilizado.

A Análise Estratégica teve como objetivo examinar o contexto da empresa e gerar informações para a concepção de solução para o PSS. Esta etapa demanda da equipe uma visão macro da realidade da empresa, da sua concorrência e de seus clientes. Observou-se como principal dificuldade a limitação dos participantes no entendimento dos elementos básicos sobre o PSS e os relacionar com questões estratégicas do negócio.

O Levantamento de Oportunidades teve como objetivo estabelecer um novo posicionamento para a empresa a partir das informações processadas anteriormente. Um problema que começa a surgir com a aplicação do método é que no mesmo são sugeridas apenas ferramentas que trabalham questões voltadas somente para relacionamentos business to costumer (B2C) e não business to business (B2B), que vem a ser o tipo de relacionamento praticado pela empresa em que foi desenvolvido o PSS.

No Projeto de Conceitos do Sistema o objetivo é detalhar a oportunidade identificada previamente. A exemplo do que ocorre na etapa de levantamento de oportunidades, o método também sugere subetapas e ferramentas que trabalham questões voltadas, prioritariamente, a relacionamentos $\mathrm{B} 2 \mathrm{C}$.

No Detalhamento do Sistema o objetivo foi detalhar os recursos do sistema para permitir a sua execução. Algumas ferramentas do método original foram descartadas por serem pertinentes para situações B2C ou voltadas para entidades sem fins lucrativos. Em algumas ferramentas propostas pelo método buscou-se um maior detalhamento das atividades a serem desempenhadas tanto pelos clientes como pelos funcionários da empresa. Em sua apresentação original não se verificava o nível de detalhamento desejado.

\section{ANÁLISE DAS INFORMAÇÕES LEVANTADAS}

As dificuldades observadas na meta-etapa de monitoramento estão organizadas na tabela 1. Algumas delas foram detectadas no levantamento citado no item 2, e estão identificadas na tabela, as demais foram verificadas com a realização deste trabalho.

Tabela 1. Listagem das dificuldades observadas no desenvolvimento do PSS. Fonte: Os autores.

\begin{tabular}{|l|l|l|}
\hline \multirow{2}{*}{$\begin{array}{l}\text { Dificuldades } \\
\text { observadas }\end{array}$} & \multicolumn{1}{|c|}{ DIFICULDADES } & \multicolumn{1}{|c|}{ COMENTÁRIOS } \\
\cline { 2 - 3 } $\begin{array}{l}\text { junto à } \\
\text { empresa }\end{array}$ & $\begin{array}{l}\text { a) Necessidade de treinamento específico da equipe } \\
\text { para o entendimento do novo posicionamento } \\
\text { estratégico da empresa; }\end{array}$ & $\begin{array}{l}\text { Dificuldade com origem na } \\
\text { falta de conhecimento sobre } \\
\text { PSS. }\end{array}$ \\
\cline { 2 - 3 } & $\begin{array}{l}\text { b) Preocupação quanto ao entendimento e aceitação } \\
\text { do novo modelo de negócios da empresa por parte }\end{array}$ & $\begin{array}{l}\text { Dificuldade prevista no } \\
\text { levantamento bibliográfico e }\end{array}$ \\
\hline
\end{tabular}




\begin{tabular}{|c|c|c|}
\hline & dos clientes; & relativa ao Marketing. \\
\hline & $\begin{array}{l}\text { c) Descrédito inicial dos funcionários em relação ao } \\
\text { sucesso do PSS; }\end{array}$ & $\begin{array}{l}\text { Dificuldade prevista no } \\
\text { levantamento bibliográfico e } \\
\text { relativa às questões } \\
\text { organizacionais.. }\end{array}$ \\
\hline & $\begin{array}{l}\text { d) Dificuldade na compreensão das mudanças para o } \\
\text { novo posicionamento da empresa; }\end{array}$ & $\begin{array}{l}\text { Dificuldade prevista no } \\
\text { levantamento bibliográfico e } \\
\text { relativa às questões } \\
\text { organizacionais.. }\end{array}$ \\
\hline & $\begin{array}{l}\text { e) Barreira cultural para o entendimento da oferta } \\
\text { combinada entre produtos e serviços; }\end{array}$ & $\begin{array}{l}\text { Dificuldade com origem na } \\
\text { falta de conhecimento sobre } \\
\text { PSS. }\end{array}$ \\
\hline & $\begin{array}{l}\text { f) Dificuldade em garantir o cumprimento das } \\
\text { atividades planejadas para o desenvolvimento do } \\
\text { PSS; }\end{array}$ & $\begin{array}{ll}\text { Dificuldade relacionada ao } \\
\text { pouco comprometimento no } \\
\text { início do processo de } \\
\text { desenvolvimento do PSS }\end{array}$ \\
\hline & $\begin{array}{l}\text { g) Desconhecimento dos conceitos básicos sobre o } \\
\text { PSS; }\end{array}$ & $\begin{array}{l}\text { Dificuldade com origem na } \\
\text { falta de conhecimento sobre } \\
\text { PSS. }\end{array}$ \\
\hline & $\begin{array}{l}\text { h) As mudanças necessárias na empresa levaram ao } \\
\text { desenvolvimento mais lento das atividades; }\end{array}$ & $\begin{array}{l}\text { Dificuldade prevista no } \\
\text { levantamento bibliográfico e } \\
\text { relativa à a cultura } \\
\text { organizacional. }\end{array}$ \\
\hline & $\begin{array}{l}\text { i) Problemas em relação ao cumprimento da agenda } \\
\text { de reuniões assim como de tarefas de } \\
\text { desenvolvimento do PSS; }\end{array}$ & $\begin{array}{ll}\text { Dificuldade relacionada ao } \\
\text { pouco comprometimento no } \\
\text { início do processo de } \\
\text { desenvolvimento do PSS }\end{array}$ \\
\hline & $\begin{array}{l}\text { j) Receio de que eventuais modificações } \\
\text { aumentariam o custo fixo da empresa; }\end{array}$ & $\begin{array}{l}\text { Dificuldade prevista no } \\
\text { levantamento bibliográfico e } \\
\text { relativa ao alinhamento } \\
\text { estratégico. }\end{array}$ \\
\hline & $\begin{array}{l}\text { k) A gestão da empresa não tinha pleno foco no } \\
\text { trabalho de implantação do PSS. }\end{array}$ & $\begin{array}{ll}\text { Dificuldade relacionada ao } \\
\text { pouco comprometimento no } \\
\text { início do processo de } \\
\text { desenvolvimento do PSS }\end{array}$ \\
\hline Dificuldades & $\begin{array}{l}\text { 1) O método utilizado não prevê a capacitação dos } \\
\text { participantes em conceitos básicos sobre PSS; }\end{array}$ & $\begin{array}{l}\text { Dificuldade com origem na } \\
\text { falta de conhecimento sobre } \\
\text { PSS. }\end{array}$ \\
\hline $\begin{array}{l}\text { observadas } \\
\text { junto ao } \\
\text { método }\end{array}$ & $\begin{array}{l}\text { m) São sugeridas ferramentas que trabalham } \\
\text { questões voltadas somente para relacionamentos } \\
\text { B2C; }\end{array}$ & $\begin{array}{l}\text { Dificuldade relativa à } \\
\text { demanda específica do } \\
\text { estudo de caso deste artigo. }\end{array}$ \\
\hline escolhido & $\begin{array}{l}\text { n) Algumas ferramentas propostas pelo método não } \\
\text { geravam o grau de detalhamento necessário para o } \\
\text { desenvolvimento do PSS. }\end{array}$ & $\begin{array}{l}\text { Dificuldade relativa à } \\
\text { demanda específica do } \\
\text { estudo de caso deste artigo. }\end{array}$ \\
\hline
\end{tabular}

Observou-se que algumas das dificuldades verificadas no desenvolvimento do PSS podem ser consideradas com a mesma origem como é o caso de "a", "e", "g" e "l" que estão relacionadas à falta de conhecimento sobre os conceitos básicos de PSS.

As dificuldades identificadas nos itens "f", "i" e "k" também têm origem comum e estão vinculadas ao pouco comprometimento no início do processo de desenvolvimento do PSS. Nos itens "m" e "n" estão as dificuldades relativas à demanda específica do estudo de caso deste artigo. 


\section{CONCLUSÃo}

Os resultados do artigo confirmam o já observado na literatura, mas também geraram contribuições. Verificou-se a existência de dificuldades não relatadas na bibliografia e referentes à preparação da empresa, mais especificamente sobre o treinamento dos seus funcionários a respeito das particularidades do Sistema Produto-Serviço. As dificuldades no processo de implantação são também causadas pelo método escolhido para o desenvolvimento do Sistema Produto-Serviço. Este resultado em específico provoca a necessidade de criação de um critério adicional no processo de seleção do método de desenvolvimento a ser empregado. Os resultados obtidos com a pesquisa contribuem para que futuros desenvolvedores de Sistema Produto-Serviço previnam-se mais adequadamente às barreiras deste processo permitindo que compreendam os problemas a serem enfrentados e preparem-se antecipadamente.

As dificuldades de implantação de Sistemas Produto-Serviço listadas aqui são particulares ao caso estudado, elas poderão se repetir ou não em outros casos em virtude do grau de particularidade observado neste processo. Outro fator de particularidade está relacionado ao método de desenvolvimento de PSS utilizado. É possível que o uso de outro método gere dificuldades distintas das observadas neste estudo de caso.

\section{REFERÊNCIAS}

BAINES, T. S. et al. State-of-the-art in product-service systems. Journal of Engineering Manufacture, v. 221, n. 10, p. 1543-1552, 1 jan. 2007.

BRAGA JR., A. E.; TOLEDO, J. C. DE. DIFICULDADES PARA O DESENVOLVIMENTO E IMPLANTAÇÃO DE UM SISTEMA PRODUTOSERVIÇOAnais do X Workshop do Instituto de Inovação e Gestão de Desenvolvimento do Produto. Anais...2016

CAVALIERI, S.; PEZZOTTA, G. Product-Service Systems Engineering: State of the art and research challenges. Computers in Industry, v. 63, n. 4, p. 278-288, maio 2012.

COUGHLAN, P.; COGHLAN, D. Action research for operations management. International Journal of Operations \& Production Management, v. 22, n. 1995, 2002.

DIMACHE, A.; ROCHE, T. A decision methodology to support servitisation of manufacturing. International Journal of Operations \& Production Management, v. 33, n. 11/12, p. 1435-1457, 2013.

GEBAUER, H.; FLEISCH, E.; FRIEDLI, T. Overcoming the service paradox in manufacturing companies. European Management Journal, v. 23, n. 1, p. 14-26, 2005.

GOEDKOOP, M. J. et al. Product Service systems, Ecological and Economic Basics. Amersfoort: Report for Dutch Ministries of Environment (VROM) and Economic Affairs (EZ), 1999.

KOHTAMÄKI, M. et al. The performance impact of industrial services and service orientation on manufacturing companies. Journal of Service Theory and Practice, v. 25, n. 4, p. 463-485, 2015.

MATHIEU, V. Service strategies within the manufacturing sector: benefits, costs and partnership. International Journal of Service Industry Management, v. 12, n. 5, p. 451475, 2001. 
VEZZOLI, C.; KOHTALA, C.; SRINIVASAN, A. Product-Service System Design for Sustainability. 1st. ed. Sheffield: Greenleaf Publishing, 2014. 